\title{
The Design and Application of Data Acquisition and Monitoring System for Laboratory
}

\author{
Rongxia Duan ${ }^{1, a}$, Baocai $X u^{2, b}$, Chunmei Wang ${ }^{3, c}$ and Ningfeng $X u^{4, d}$ \\ ${ }^{1,2}$ Ordnance Engineering College,Shijiazhuang, China \\ ${ }^{3}$ Hebei University of Science \& Technology, Shijiazhuang, China \\ ${ }^{4}$ Ningbo Institute of Technology, Zhejiang University, Ningbo, China

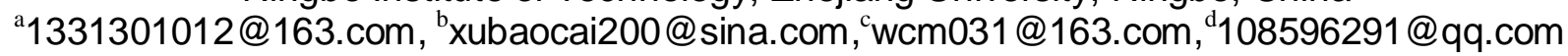

Keywords: Data collection; Laboratory; Monitor and control system; Design; Application

\begin{abstract}
Laboratory data acquisition monitoring system is designed for a school laboratory developed, solved about the problems such as data acquisition, real-time monitoring, alarm, greatly improving the work efficiency, and improve the accuracy of the testing data and real-time performance. This paper expounds the design and development of the system from both hardware and software. The hardware part adopts the overall linear network topology, which mainly includes the collection module, the hub module, the network interface configuration module, the monitoring computer and so on. Software parts are developed in VB.NET, including interface and data transfer programs, system patrol procedures, data logging programs, and device status checks. The system implements the next function; The test data is collected in real time, which is the system patrol function, the system can detect the test data of each remote terminal in real time, and display the monitoring interface. (2) test abnormal alarm, if detection in the process of test data over the upper or lower limit set by the system will give alarm prompt, and the monitoring interface corresponding entry will be red, prompt test personnel timely treatment; (3) test data record, including the entry of the test sample record, the experiment data of the basic information, alarm information records;(4)test information processing, automatic processing system also implements the test time and automatic generation of test single function. The system function verification results show that the system meets the design expectation. This laboratory data collection monitoring system has been used for user testing, which can greatly reduce the staff's workload. At the same time, the system is of reference to the data collection monitoring management of other similar laboratories.
\end{abstract}

\section{Introduction}

Industries in recent years are used for the reliability of the electronic components put forward higher requirements, more and more reliability test tasks, test equipment more and more, laboratory space, are growing, traditional monitoring mainly depends on artificial field patrol, observation record the experiment data, send will need to invest a lot of human to live every day patrol inspection and record data, this management mode has restricted the laboratory management efficiency; In addition, about the exception handling, even if most of the test chamber has own transfinite alarm device, but as a result of lab area is large, can't hear alarm equipment in the office, if the alarm occurs in the clearance between the personnel on duty patrol, patrol personnel cannot discover the abnormal test, can't timely, which may lead to test failure sample, abnormal results, etc. Traditional by artificial test data collection and monitoring methods cannot satisfy the needs of business development laboratory, how to hundreds of units at the same time open the chamber for the timely and effective monitoring, how to record the mass of the test data, storage, processing, one is an urgent need to solve the problem. To sum up, the laboratory needs an automated data collection monitoring system. All the staff on duty in the office can be observed in the operation condition of the test equipment, including parameters such as temperature, humidity, voltage, current, and record test data, analysis of experimental data. System can real-time monitor the test status, test information feedback, greatly improved work efficiency, reduce the probability of error 
at the same time, to a certain extent will be monitoring personnel from heavy patrol inspection, data records, sorting, analysis, liberated[1-2].

\section{The Hardware Design and Implementation Plan}

The hardware system consists mainly of power supply, sensor, signal acquisition circuit, single chip, PHY and network connection. The power source module produces $-5 \mathrm{v}$ voltage and $+3 \mathrm{~V}$ voltage through the power source module. The temperature sensor chooses the PT1000, the MSP430 for the single chip, the DP83848C for the PHS, and the Internet connection to the rj-45 with the network transformer, the RS232 and RS485.

Due to the wide distribution of laboratory equipment, the transmission line is longer, so the RS485 interface is long and reliable and stable. The connection between the hub and the monitor computer is a low cost, easy to connect RS232 interface. Specific connection scheme is: PT1000 humidity sensor by RS485 interface connected to the collector, between the acquisition module and a hub, hub and RS485 interface connection, between a hub and monitor computer with RS232 interface to connect.

RS232 agreement, RS232 interface on the bus is only allowed to connect a transceiver equipment, and RS485 communication connection online for a maximum of 32 sets of equipment, the multibus architecture is adopted, which has four bus acquisition module, on each bus to connect up to 10 acquisition module. With separate dialing address, each hub can be matched up to 40 signal collection modules to complete the signal collection processing in 10 regions. At the same time, each hub and other hubs form the RS485 network to enable communication between hubs. This makes up the network topology. The network topology structure is shown in Fig.1.

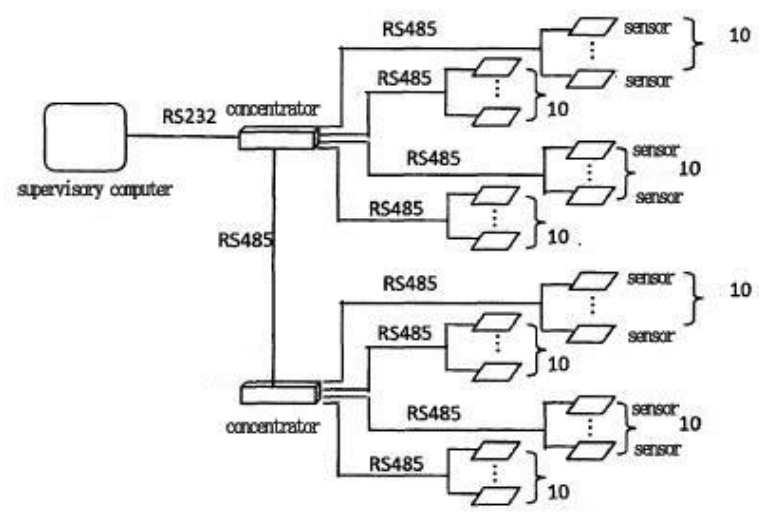

Figure 1. Network topology diagram

The design of this system is combined sensor examination and single chip control, through the single chip computer are connected to the sensor, data acquisition and storage of sensor detection, on the other hand with monitor computer through the RS - 232 bus communication, the collected data to monitor and control computer. Monitor the data that the computer will receive to record, store, process, for the staff to browse, record and the relevant processing.

\section{The System Software Design Scheme}

In the software aspect, the $\mathrm{H}$ layer $\mathrm{B} / \mathrm{S}$ design is adopted, which mainly includes the business presentation layer, the logical layer, and the data access layer. When the requesting user interface layer, logic layer from the presentation layer call the corresponding method for database operations, and then the data results returned to the user operation interface layer. The sample user interface layer does not need to know the structure of the database, as long as it ensures that the interface between the business layer is normal. Deliver, do not contact directly with the database user interface layer, increase the safety of the database to a certain extent, to the user interface layer developers also reduces the requirement. System is the use of the network interface configuration module and data acquisition module method is simple, the user can easily operate, background 
management difficulty is not high also, through a simple training in a short period of time also can easily get started. System software can also be implemented in modular design. The system software consists mainly of the database, the inspection and the recording procedure, the alarm procedure, the test information processing program and so on.

Data acquisition module design.The data collection module USES the RS232 interface and Modbus protocol for data collection and transmission. The Modbus protocol is a popular protocol for the industry and supports the devices of RS232, RS422, RS485 and other interfaces. Considering the type of laboratory equipment, most of the old equipment has no corresponding interface, and the Modbus protocol is chosen for the new generation after the convenience test equipment $\mathrm{W}$. The design of the data collection module interface includes the principles of the Modbus protocol, the calculation of the crc-16 calibration code, and the compilation of data transfer functions.

Modbus protocol works: defines the data structure, commands, and messages, in a way, data communication with the method of master-slave, data from a primary end request message, from a terminal receives the right message to send data to the main response to the request, the client also can send messages directly from the modified data, realize the two-way, speaking, reading and writing. When communication command sent to the equipment, in accordance with the corresponding address code equipment communication command, and remove the address code, read the information, if there is no error, the corresponding tasks; The execution result is then sent back to the sender. Foldback information includes address code, function code to perform an action, to perform an action after the results of data and error checking code, if error will not send any information.

CRC - 16 basic principle is: suppose that there are information code of $\mathrm{K}$, the $\mathrm{K}$ bit information code again after joining together $\mathrm{R}$ a check code, the code length is $\mathrm{N}$, as a result, the code also called $(\mathrm{N}, \mathrm{K})$ code. For a given $(\mathrm{HK})$ code, to prove that there exists a maximum time act of $\mathrm{N}-\mathrm{K}=$ R polynomial $G(X)$, based on $G(X)$ can generate $K$ bit information check code, and $G(X)$ is called the CRC code generating polynomial.

Specific check code generation process for: suppose send information with polynomial $\mathrm{C}(\mathrm{x})$, said the move $\mathrm{C}(\mathrm{x})$ left $\mathrm{R}$, can be represented as $\mathrm{C}(\mathrm{x}) * 2 \mathrm{R}$, this will empty $\mathrm{C}(\mathrm{x})$ on the right side of the $\mathrm{R}$, this is the location of the check code. The remainder of $\mathrm{C}(\mathrm{x}) * 2 \mathrm{R}$ divided by the resulting polynomial $\mathrm{G}(\mathrm{x})$ is the check code.

The crc-16 error detection is done using the division and the remainder principle, and the crc-16 contains two bytes, or 16 bits, and passes 8-bit characters. The crc-16 is computed by the sending device, placed at the end of the sending message. Receive information of equipment to calculate again to receive information of CRC - 16 yards, comparing calculated CRC code is consistent with the received, if the two is not consistent, is wrong.

CRC - there are two methods to calculate 16 yards, a look-up table method, the advantage is the small amount of calculation, quick speed, its shortcomings are much module code, modify the inflexible, poor portability. One is the calculation method, which is based on the production principle of the crc-16 check code. Compared to the lookup table method, the advantages are less module code, flexibility, and portability. The drawback is that the calculation is large.

The main design steps are as follows:

1) define functions and variables as follows:

Public Sub BuidCRCTab()

Dim intl,iniJ As Integer

Dim bytnPolyLo As Byte $=\& H 1$, bytPolyLyHi As Byte $=\&$ HAO

Dim bytTempLo As Byte, bytTempHi As Byte

Dim bytCRCLo As Byte, bytCRCHi As Byte

2) the code statement that generates a single byte is as follows:

For intI $=0$ To 255

bytCRCLo=CByte (intI)

bytCRCHi $=0$

For int $\mathrm{J}=0$ To 7

bytTenpLo=bytCRCLo 


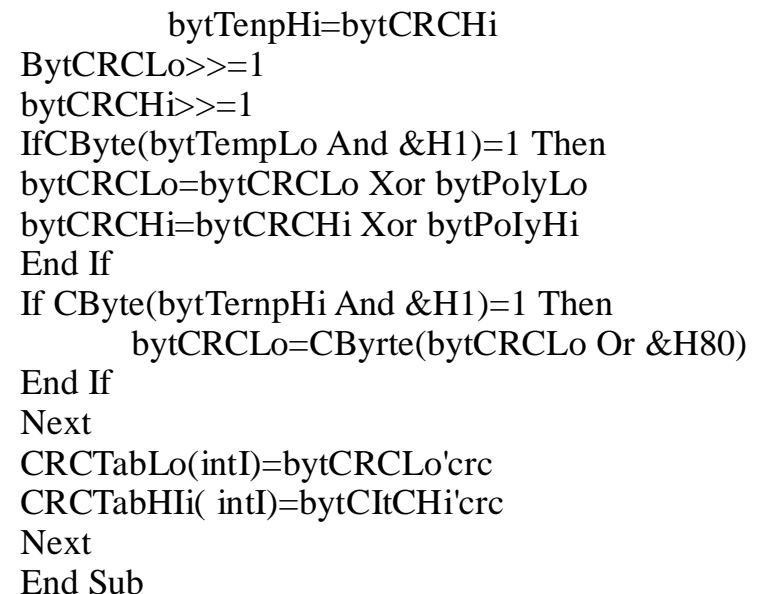

Application Software Design Presentation. System is mainly divided into the inspection and record (data acquisition and recording), test information processing two big function module. Inspection and record module can realize system inspection and equipment state discriminant functions, including system inspection record function can be realized in the equipment of experiment data acquisition and state real-time monitoring, can also in abnormal situation such as the data of the test process of transfinite alarm, at the same time, system to record data collected can also store; The experimental information processing module includes automatic test time and automatic test form. Fig. 2 is the system function diagram.

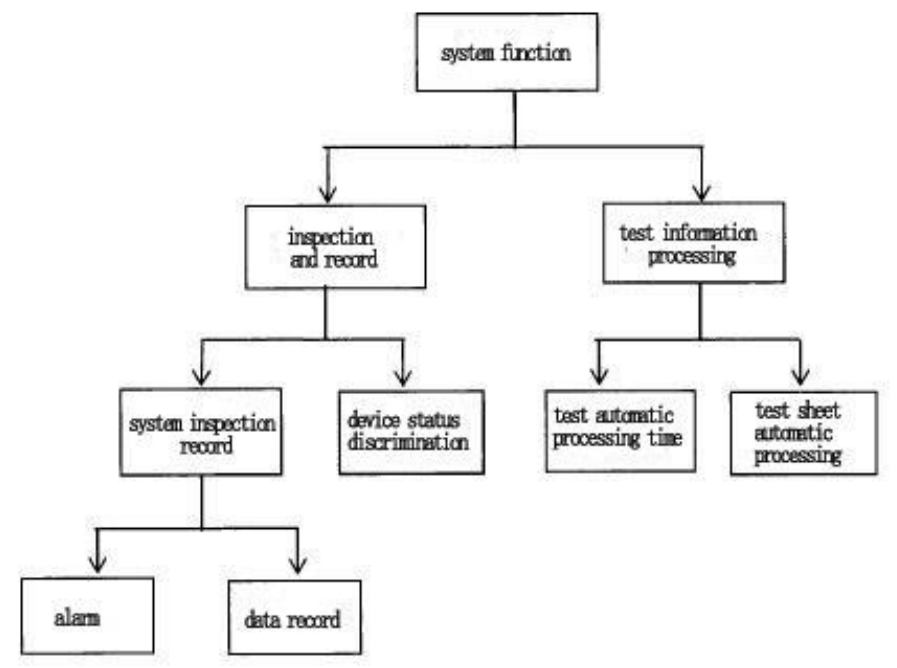

Figure 2. system function diagram

Checking and Recording Module Design. System checking function is mainly to various online equipment inspection, real-time monitoring the equipment running status, the input and the system to record store data collected, including the test model of a single number, name of the sample, sample quantity, test items, test conditions, test equipment, test, shots of test time, sampling, sampling time and other relevant information and test records, found that more than test set conditions timely alarm, alarm is shown as: sends an alarm sounds, alarm equipment icon, display value to become red, equipment below arrow appears behind the temperature value of the upward or downward (humidity is lower than the set limit to show the "left", the temperature is higher than the set time limit on display "write"). And will alarm information record into the database, including basic information at equipment information, alarm, batch sample (test number, name of the sample model, etc.), alarm time, alarm, data content, data content display alarm temperature, the amount is also show alarm temperature exceeding the maximum limit temperature or more than the lower limit. As an archive, it is convenient for the data to be reviewed and analyzed. The inspection module design process is shown in Fig.3. 


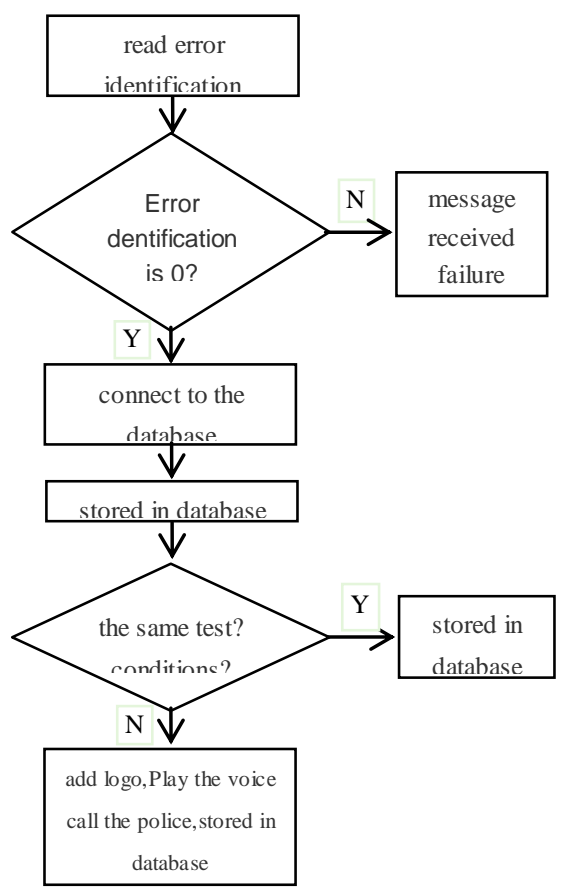

Figure 3. inspection and record module flow chart

Test Information Processing Module Design. The experimental information processing module includes test time automatic processing and test sheet automatically processing two parts. Test time automatic processing is to prevent the time calculation error, user input is required to test the start time, the system automatically calculate according to the test cycle test end date and specific time. Design idea: second calculation for the unit test time, from the text box for test information and the current system time, statement of each variable for time calculation, test time and 24 do integer modulo operation, test the remaining days respectively and the remaining time, turning over time to convert hours, get the final test duration. The automatic processing function is an Excel spreadsheet instead of a paper test. Design idea is: the definition of Excel application interface, connect to the database and read from the DataCheck8 form RunTime1, RunTime2, CheckTime column values, set the cell properties, and automatically written to the related content, finally save the file.

\section{Conclusion}

This article developed the system from two parts of hardware and software. The hardware part adopts the overall linear network topology, which mainly includes the collection module, the hub module, the network interface configuration module, the monitoring computer and so on. Software part use VB.NET for development, development of each functional module, including interface and data transmission program, system inspection, data record program, equipment status discrimination, etc. System function verification results show that the system meet the design expectations, realizes the testing data real-time acquisition, namely the system inspection function, system can get the real-time detection of test data from a remote terminal, displayed on the monitor interface. If in the process of implementation the abnormal alarm test, the test data over the upper or lower limit set by the system will give alarm prompt, and the monitoring interface corresponding entry will be red, prompt test personnel handle in time. Implements test data record, including the entry of the test sample record, the experiment data of the basic information, alarm information records, etc., the above data are recorded into the system database, in case of late query, invoke, analysis, etc.; The automatic processing of experimental information was realized, and the system also realized the automatic processing and auto-generated experiment. The system/user trial, greatly reduce the workload of staff, improve the working efficiency and test response capabilities, the laboratory from manual management to the key to the development of intelligent management.

\section{References}

[1] A. Neto, H. Fernandes, A. Duarte, Firesignal-Data acquisition and control system FusionEngineering and Design2007.82 1359-1364.

[2] Serizawa,YTanaka,T.Yusa,H.Koda,YYamashita., Verification of Distributed real-Time Computer Network Architecture Associated With Off-The-Shelf and Dedicated Technologies.IEEE Transactions on Power Delivery.EISCI58(3).2009. 
[3] Alan Stevens.Microsoft SQL Server2005 Express.Personal Computer Wor1d.EI2006 7.

[4] What You Need to Know About Microsoft SQL Server2008R2.PAUL THURROTT《Windows IT Pro 》.2010.1

[5] Tang Yu, Peng Hu. Ultrasound bone density measurement system design [J]. Biomedical Engineering Research, 2006,25 (4) :224-232.

[6] Song Guangde, Hu Hongbo. FPGA-based digital flaw detection system A super study [J]. Electronic Technology Application, 2003 (10):29-31.

[7] Sun Air. Xilinx programmable logic devices, advanced applications and design techniques [M]. Beijing: Electronic Industry Press, 2004.

[8] ADLINK Technology.PCI-9111 DG / HR multi-functions data acquisition card User's guide [EB / OL]. Http://www.adlinktech.com/PD/Download/adlinktechnology-index.php.2008-01- 10.

[9] Wu Ontario, Tai Ming, the Tao. Windows 2000/XP WDM device driver development [M]. Beijing: Electronic Industry Press, 2003.

[10] PLX Technology, PCI 9052 Data Book [EB / Http://www.plxtech.com/download/PCI9000/9052/databook/9052db-20.pdf.2008-01-10.

[11] Liu Jun, Xiao Zi Xiang. Driver program to share data with users in several ways [J]. ITS APPLICATIONS, 2002, 21 (6): :-11.

[12] Liu Chuanyong, Zhang, Blow, et al. Two-center overlap Spinal Treatment Machine [J]. Biomedical Engineering Research, 2006,25 (1) :55-57 